\title{
FACTORS AFFECTING THE POPULATION FLUCTUATIONS OF TERRESTRIAL SNAILS Monacha cantiana (MÜLLER) AND Succinea putris (LINNAEUS) AT FARASKOUR DISTRICT, DAMIETTA GOVERNORATE \\ Awad, M. H. M. ; M. A. Osman and A. H. Hamad \\ Plant Protection Research Inst. Agric. Res. Center, Egypt Zoology Dept. Agri. Coll. Mansoura university
}

\begin{abstract}
These studies were carried out at Faraskour district in four locations differed in its soil such as Sharabass which located very closely to Nile river coast with fine loamy clay soil and highly infested with land snails to El-Rodda with heavy clay soil reclaimed from Manzala Lake. Ecological factors were studied by measuring the population in moist and dry soil in the same village under all field conditions. Also, toxicological studies were conducted on these land snails under field conditions to know, the best compound which effective and cheaper in addition to avoid environmental pollution and harmful residues on plants, animal and human health. Tested compounds stated that superphosphate mixed with hyderated lime by percentage of 3:1 and foliar fertilizer improved plant growth and quality in addition to limiting individuals of these pests in relatively similar effect of some pesticides. Data showed high differences between moist and dry soil which recorded (64.6/34.8), (35.5/15.9), (35.8/18.0) and (38.5/17.4) respectively, also stated that high recorded individuals in moist soil of clover fields (64.8 per $\mathrm{m}^{2}$ at sharabass).
\end{abstract}

\section{INTRODUCTION}

In recent years, land snails became very important pests to field, horticultural and ornamental plants and caused great damage not only to plants but also animal, bird and human health, in addition acting as intermediate hosts of parasitic worms such as Fasciola, Monizea, Shistosoma and nematodes. Also, spread fungal, bacterial and viral diseases, Godan (1983), Bishara et al. (1968), and El-Okda (1979), (1984) who reported about these pests, their dispersal, activity, economic importance and their control by pesticides or plant extracts. Many investigators reported about their biology, ecology and control, Hashem et al. (1993), and Ghamry et al. (1991). The present work aimed to find the best and suitable way for controlling these pests without chemical pesticides which caused environmental pollution and harmful for human and animal health due to residual effect in field, vegetable and fruit crops which treated with these pesticides. So, fertilizers such as superphosphate mixed with hydrated lime and Foliar fertlizer were used as tested compounds against these land snails to study their effect in comparison with Metaldhyde bait as recommended molluscicides . 


\section{MATERIALS AND METHODS}

\section{1- Population dynamic of land snails:}

a) Population of individuals measured at four locations of Faraskour district by counting individuals in four types of soil differed from moist and dry loamy sand to heavy clay soil. Also, samples were taken from two types of soil, numbers from wet soil collected after 5 days from irrigation while the other collected 15 days after irrigation.

b) Seasonal fluctuation of snails was measured through autumn, winter and spring of (2006/2007), Godan (1983), Port and Ester (2002), and Satoshi (2003).

2- Land snail control:

Control under field condition divided into three treatments two of them were fertilizers such as:

a) Superphosphate $15 \%$ provided by agricultural fertilizers (Abo Qure Factory for Fertilizers) mixed with hydrated lime by percentage of 3:1. (100Kg / feddan)

b) Foliar fertilizer which contains coppersulphate manganese, zinc and phosphore in sort of aquatic case (one litre/ fedan), according to Kloos and Cullough (1982).

c) Metaldhyde bait in recommended dose provided by Kafr ElZayyat for insecticides used in special bait station designed to avoid pollution Godan (1983).

3- Technique used:

a) Samples from field and vegetable crops in three successive seasons which recorded snail's activity through autumn, winter and spring of (2006/2007), Godan (1983), El-Okda (1979, 1980, 1981 and 1984), Hashem et al. (1993). these samples of snails population were taken from clover, wheat, patata and lettuce per $\mathrm{m}^{2}$ for each crop and five replicates for each sample from moist and dry soil monthely, according to Godan, (1983).

b) Control measurement:

Three treatments of control were considered as follows:

- Using superphosphate $15 \%$ mixed with hydrated lime (3:1) as dust on treated plants in plots of $1 \mathrm{~m}^{2}$ for each replicate. (100kg/feddan)

- Foliar fertilizer as spry on treated replicates. (one litre / feddan)

- Metaldhyde bait stations in the same area of treated replicates. $(2 \mathrm{~kg} /$ feddan)

- Affected individuals collected and counted according to Henderson and Tilton formula, (1925) and corrected by Abott (1925).

\section{RESULTS AND DISCUSSION}

Data obtained in table (1) showed that, samples of individuals which collected from field and vegetable crops such as clover, wheat, patata and lettuce showed high variation between moist and dry soil were recorded (64.6/34.8), (35.5/15/9), (35.8/18.0) and (38.5/17.1) for (moist/dry) in clover, 
wheat, patata and lettuce infested by $M$. cantiana at Sharabass coast of Faraskour district. Also, Faraskour city, El Dahra village and El-Rodda city showed that the data obtained from these locations were similar to that obtained from Sharabass coast, the highest number of individuals was in clover moist soil at Sharabass and El-Dahra villages which recorded (64.8 and 62.0), respectively. While, the lowest number was recorded in wheat dry soil (15.9) followed by dry soil of lettuce at Faraskour city (14.6). Wet or moist soil showed high number of individuals in most tested locations which agreed with that reported by Godan (1983) and El-Okda (1979, 1980). Also, the highest number was recorded in clover in most samples of studied locations, which recorded $(64.6,62.0,57.9$ and 57.1) at Sharabass in clover moist soil at El-Rodda village by lettuce at the same village 50.6 followed by patata which recorded (49.8 and 48.9) at El-Rodda and Faraskour, respectively. It was noticed that land snail Succinea putris was more affected by soil moisture than $M$. cantiana which showed more resistance for dryness that also affected by the shelter of plants such as in case of clover makes soil moist and warm for long time followed by potato, lettuce and finally wheat. This shelter for these snails agreed with that reported by Godan (1983), ElOkda (1980), Satoshi (2003), Duncan (1983) and Coote (2003).

\section{2- Land snail control:}

\section{a- Control of S. purtis}

Three tested compounds were used against land snails under field conditions. Data of table (2) indicated that metaldhyde was more effective in each of lettuce with $85 \%$ reduction on S. putris followed by wheat $83 \%$ at ElDahra village. Also, on patata and wheat at El-Rodda city $80 \%$ recorded for each. Its efficiency on lettuce $77 \%$ and $75 \%$ on patata but in most replicates was similar or closely to the result of tested fertilizers, foliar fertilizer recorded $71 \%$ reduction in snail individuals on lettuce at Faraskour followed by $69 \%$ on patata at El-Dahra village and in lettuce and clover $68 \%$ for each. It was observed that locations which treated with Foliar and Superphosphate improved clover and it was clear on lettuce, patata and wheat, respectively beside its efficiency on individuals of $S$. putris which agree with that reported by Godan (1983) and Marston and Hostetmann (1985).

\section{b) Control of M. cantiana}

Tested compounds were less effective than in $S$. putris as shown in table (3) which stated that Metalhdhyde bait recorded satisfied results in all cases of reduction percentages after 15 days of treatment $81,79,76$ and 75 $\%$ on potato, wheat and lettuce at El-Rodda, El-Dahra, El-Rodda and Faraskour, respectively. The lower effect of metaldhyde was on individuals under clover crop $63 \%$ and $64 \%$ at Sharabass and Faraskour, respectively. Also, mean of reduction percentages due to the effect of superphosphate and foliar were less than in S. putris specially on clover plant where recorded 50 $\%$ and $53 \%$ at Sharabass, respectively. 
Awad, M. H. M. et al.

T1 
J. Agric. Sci. Mansoura Univ., 34 (2), February, 2009

T2-3

1325 
The highest effect of superphosphate was on lettuce $69 \%$ at Sharabass followed by $64 \%$ on lettuce at El-Dahra. Clover plant showed less efficiency for tested compounds due to the heavy plants of clover as shelter save protection and recovery to land snails also clover as asuitable for hidden and food sources, that agree with that investigated by Godan (1983) and Kloos, (1982).

\section{REFERENCES}

Abbott, W. S. (1925). A method of computing the effectiveness of an insecticides. J. econ. Entomol-18: 265-267.

Bishara, S. I., M. S. Hassan and A. S. Kalliny (1968)studies on some land snails injries to agriculture in U.A.R Rev. Zool. Bot., Agric., Ixxvll (3-4) : $239-252$.

Coote T., E. Loeve (2003). From 61 species to five: endemic tree snails of the society islands fall prey to an ill-judged biological control program. Oryx $37,91-96$.

Duncan, J. (1983). The biological and physiological basis of the mode of action of molluscicides. "Plant molluscicides, E. M. Kenneth, ED", John Wily \& Sons Ltd pp 27-44.

El-Okda, M. M. K. (1979). Land snails of economic at Alexandria region with some notes on the morphological features, classification, economic damage and population density on ornamental plants. Agric. Res. Rev., $57(1): 125-130$.

El-Okda, M. M. K. (1980). Land snails of economic importance on vegetable crops at Alexandria and neighboring regions. Agric. Res. Rev., 58 (1): 49-85.

El-Okda, M. M. K. (1984). Land mollusca infestation and chemical control in Ismailia Governorate. Agric. Res. Rev., 62 (1): 87-92.

Ghamry, E. M.; H. I. El-Deeb and H. Abdel Al (1993). Efficiency of certain pesticides against some land snails under field conditions of Sharkia Governorate. Egypt. J Appl. Sci., 8 (6): 764-774.

Godan, D. (1983). Pest Slugs and Snails: Biology and Control Springer, Verlag, Berlin, Heidelberger. 445pp.

Hashem, A. G.; J. M. Nakhla, A. W. Tadros and M. A. Korashy (1993). Monitoring land snails on sweet orange trees in Beheira Governorate, Egypt, Zagazig. J. Agric. Res. 20: 691-698.

Henderson, G. F. and E. W. Tiliton (1955). Test with acaricides against the brown wheat mite. J. Econ. Entomol., 84: 157-161.

Kassab, A. and H. Daoud (1964). Notes on the biology and control of economic importance land snails in U.A.R. Agric. Res. Rev., 42: 77-98.

Kloos, H. S. F. Mc Cullough (1982). Plant molluscicides. J. Med. Plant, Res. (Planta Medica) 46, 195-209.

Marston, A. and K. Hostetmann (1985). Plant molluscicides. phytoche-mistry. $24,639-652$. 
Port, G.and A. Ester (2002). Gastropods as pests in vegetable and ornamental crops in western Europe J. molluscs as crop pests. Edited by G. M. Barker CABI Publishing Wallingford, U. K.

Satosh, C. (2003). Species Diversity and conservation of Mandarina an Endemic land snail of the Ogasawara islands. JP/Publication/Ger. PDF/ 07/01-04 PDF.

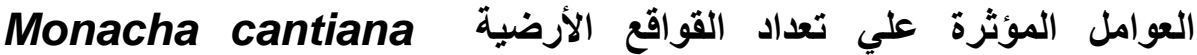 في مركز فارسكور (Müller) and Succinea putris (Linnaeus)}

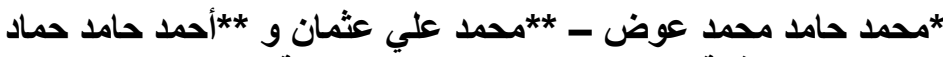

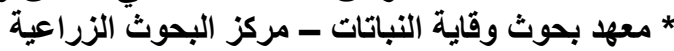

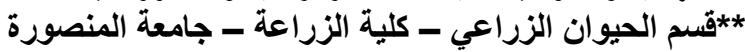

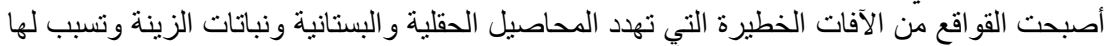

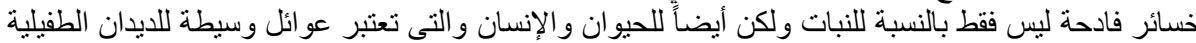

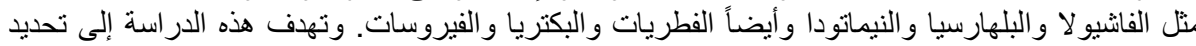

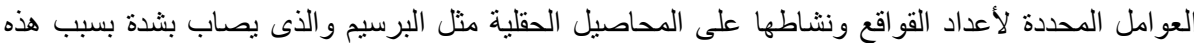

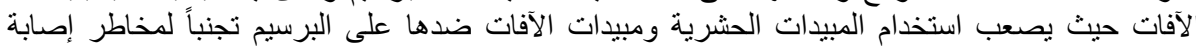

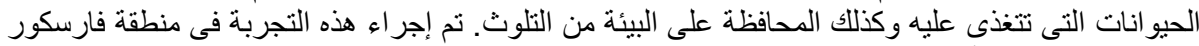

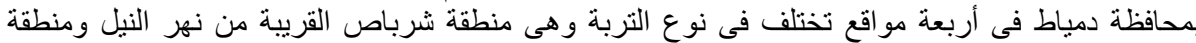

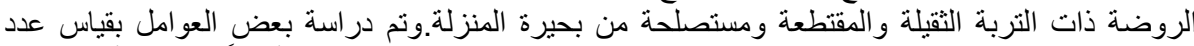

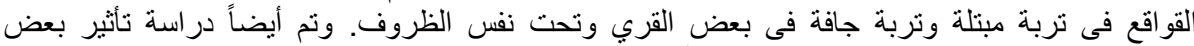

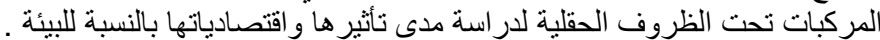

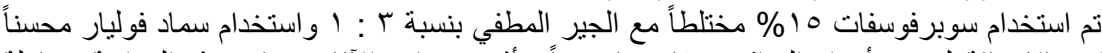

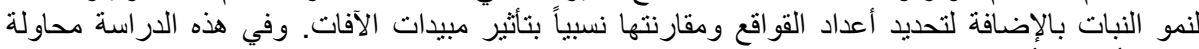

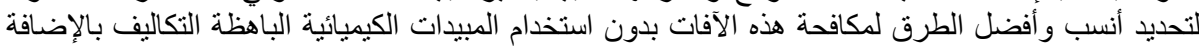

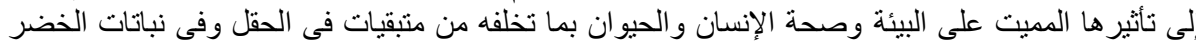

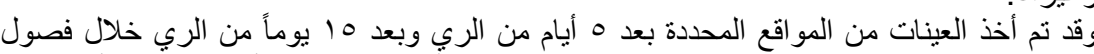

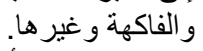

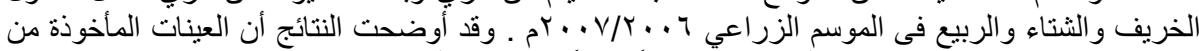

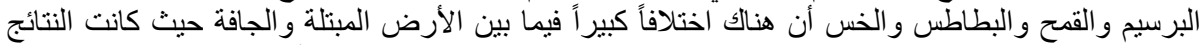

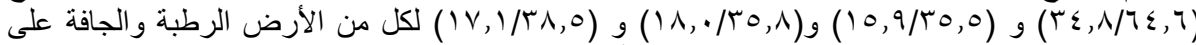

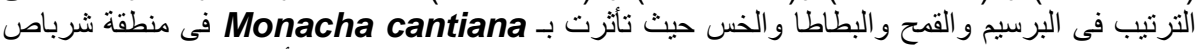

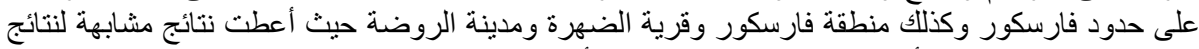

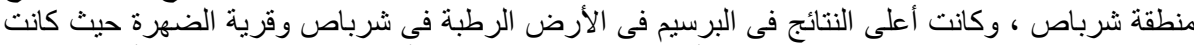

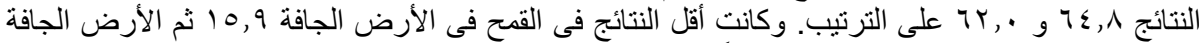

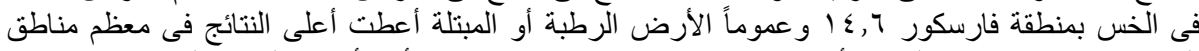

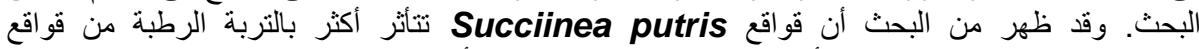
Monacha cantiana

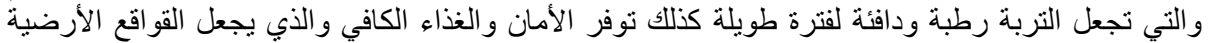

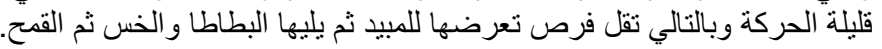

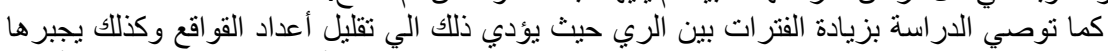

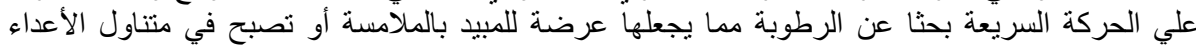
الحيوية التي تتخذي عليها. 
Awad, M. H. M. et al. 
Table 1: Population fluctuation of Monacha. cantiana and Succinea. putris on field and vegetable crops through two successive seasons in different locations at Faraskour district, Damietta Governorate

\begin{tabular}{|c|c|c|c|c|c|c|c|c|c|c|c|c|c|c|c|c|c|c|c|}
\hline \multirow{4}{*}{ Location } & \multirow{4}{*}{ Seasons } & \multicolumn{16}{|c|}{ Number of individuals of } & \multirow{3}{*}{\multicolumn{2}{|c|}{$\begin{array}{l}\text { Climatic } \\
\text { Factors }\end{array}$}} \\
\hline & & \multicolumn{8}{|c|}{ M. cantiana } & \multicolumn{8}{|c|}{ S. putris } & & \\
\hline & & \multicolumn{2}{|c|}{ Clover } & \multicolumn{2}{|c|}{ Wheat } & \multicolumn{2}{|c|}{ Potato } & \multicolumn{2}{|c|}{ Lettuce } & \multicolumn{2}{|c|}{ Clover } & \multicolumn{2}{|c|}{ Wheat } & \multicolumn{2}{|c|}{ Potato } & \multicolumn{2}{|c|}{ Lettuce } & & \\
\hline & & Moist & Dry & Moist & Dry & Moist & Dry & Moist & Dry & Moist & Dry & Moist & Dry & Moist & Dry & Moist & Dry & ${ }^{\circ} \mathrm{C}$ & $\begin{array}{c}\mathbf{R H} \\
\%\end{array}$ \\
\hline \multirow{3}{*}{ (1) } & Au. & 68.8 & 34.2 & 36.4 & 12.8 & 32.4 & 18.8 & 36.2 & 20.4 & 34.4 & 18.2 & 40.8 & 26.0 & 42.6 & 24.2 & 34.4 & 18.6 & 22 & 68 \\
\hline & Wi. & 40.6 & 21.6 & 22.0 & 8.0 & 28.4 & 10.4 & 30.4 & 12.2 & 20.8 & 6.4 & 30.4 & 14.4 & 27.8 & 9.8 & 14.6 & 7.8 & 18 & 58 \\
\hline & Sp. & 84.6 & 48.6 & 48.0 & 26.8 & 46.6 & 24.8 & 48.8 & 18.6 & 66.8 & 28.4 & 48.4 & 28.0 & 54.4 & 24.6 & 48.2 & 18.8 & 24 & 64 \\
\hline \multicolumn{2}{|c|}{ Total } & 193.8 & 104.4 & 106.4 & 47.6 & 107.4 & 54.0 & 115.4 & 51.2 & 122.0 & 53.0 & 119.6 & 68.4 & 124.8 & 58.6 & 97.2 & 45.2 & & \\
\hline \multicolumn{2}{|c|}{ Mean } & 64.6 & 34.8 & 35.5 & 15.9 & 35.8 & 18.0 & 38.5 & 17.1 & 40.7 & 17.7 & 39.9 & 22.8 & 41.6 & 19.5 & 32.4 & 15.1 & & \\
\hline \multirow{3}{*}{ (2) } & Au. & 56.4 & 26.6 & 44.8 & 24.6 & 38.2 & 18.2 & 34.6 & 14.8 & 48.8 & 28.4 & 36.8 & \begin{tabular}{|l|}
16.4 \\
16.4 \\
\end{tabular} & 48.2 & 20.4 & 34.6 & 14.6 & 22 & 64 \\
\hline & Wi. & 36.6 & 18.8 & 32.6 & 12.6 & 24.4 & 19.4 & 22.4 & 8.8 & 34.8 & 12.6 & 24.2 & 8.6 & 36.0 & 16.0 & 26.8 & 8.8 & 19 & 56 \\
\hline & Sp. & 78.2 & 38.4 & 70.6 & 40.8 & 54.6 & 14.4 & 48.6 & 18.6 & 64.2 & 24.8 & 52.2 & 24.8 & 62.4 & 32.4 & 64.6 & 24.6 & 26 & 68 \\
\hline \multicolumn{2}{|c|}{ Total } & 171.2 & 83.8 & 148.0 & 77.8 & 117.2 & 52.0 & 105.6 & 42.2 & 147.4 & 65.8 & 113.2 & 49.8 & 146.6 & 68.8 & 126.0 & 48.0 & & \\
\hline \multicolumn{2}{|c|}{ Mean } & 57.1 & 27.9 & 49.3 & 25.9 & 39.1 & 17.6 & 35.2 & 14.6 & 49.1 & 21.9 & 37.7 & 16.6 & 48.9 & 22.9 & 42.0 & 16.0 & & \\
\hline \multirow{3}{*}{ (3) } & Au. & 58.6 & 28.8 & 46.4 & 28.2 & 38.4 & 18.6 & 36.2 & 18.0 & 48.6 & 29.8 & 34.4 & 10.4 & 42.8 & 18.6 & 32.0 & 12.8 & 20 & 64 \\
\hline & Wi. & 46.4 & 26.4 & 32.8 & 14.4 & 24.2 & 10.8 & 28.4 & 10.0 & 26.4 & 14.8 & 18.8 & 12.0 & 28.6 & 10.4 & 18.8 & 8.4 & 18 & 66 \\
\hline & Sp. & 82.0 & 34.0 & 64.2 & 28.4 & 48.8 & 26.4 & 54.6 & 24.6 & 64.8 & 28.4 & 48.2 & 18.0 & 58.4 & 28.6 & 48.2 & 21.2 & 24 & 58 \\
\hline \multicolumn{2}{|c|}{ Total } & 186.0 & 89.2 & 143.4 & 81.0 & 111.4 & 55.8 & 119.2 & 52.6 & 139.6 & 73.0 & 101.4 & \begin{tabular}{|l|}
40.4 \\
\end{tabular} & 129.8 & 57.6 & 99.0 & 42.8 & & \\
\hline \multicolumn{2}{|c|}{ Mean } & 62.0 & 29.7 & 47.8 & 27.0 & 37.1 & 18.6 & 39.7 & 17.5 & 46.5 & 24.3 & 33.8 & \begin{tabular}{|l|}
13.4 \\
\end{tabular} & 43.3 & 19.2 & 33.0 & 14.3 & & \\
\hline \multirow{3}{*}{ (4) } & Au. & 56.6 & 28.4 & 48.4 & 26.8 & 46.4 & 18.6 & 48.2 & 28.4 & 42.2 & 22.2 & 40.8 & 20.8 & 56.4 & 28.4 & 46.6 & 18.8 & 21 & 62 \\
\hline & Wi. & 36.4 & 12.2 & 36.2 & 11.4 & 34.2 & 14.0 & 32.4 & 18.6 & 28.4 & 18.0 & 26.4 & 8.6 & 24.2 & 8.6 & 24.4 & 10.2 & 19 & 58 \\
\hline & Sp. & 80.6 & 34.4 & 68.8 & 22.8 & 66.8 & 26.6 & 68.6 & 22.4 & 86.8 & 46.2 & 64.2 & 41.3 & 68.8 & 28.2 & 80.8 & 42.6 & 26 & 64 \\
\hline \multicolumn{2}{|c|}{ Total } & 173.6 & 75.0 & 153.4 & 61.0 & 146.4 & 59.2 & 149.2 & 69.4 & 167.4 & 86.4 & 131.4 & 70.7 & 149.4 & 65.8 & 151.8 & 71.6 & & \\
\hline \multirow{2}{*}{\multicolumn{2}{|c|}{ Mean }} & 57.9 & 25.0 & 51.1 & 20.3 & 49.1 & 19.7 & 49.7 & 23.1 & 55.8 & 28.8 & 43.8 & 23.3 & 49.8 & 21.9 & 50.6 & 23.9 & & \\
\hline & & 2. & 46 & 4.2 & & 2.3 & & 2.4 & & 2.2 & & 4.4 & & 2.4 & & 2.1 & & & \\
\hline
\end{tabular}

Keyword: (1) = Sharabass, (2) = Faraskour, (3) = El-Dahra, (4) = El-Roda, Au= Autumn, Wi=Winter, Sp=Spring

Keist = Moist soil sampled 5 days after irrigation, $\quad$ Dry= Dry soil sampled 15 days after irrigation
Mois 
Table 2: Effect of tested compounds against Succinea putris on certain field and vegetable crops at Faraskour district, Damietta Governorate

\begin{tabular}{|c|c|c|c|c|c|c|c|c|c|c|c|c|c|}
\hline \multirow{3}{*}{ Location } & \multirow{3}{*}{$\begin{array}{c}\text { Tested } \\
\text { compounds }\end{array}$} & \multicolumn{12}{|c|}{ Number of affected individuals of Succinea Putris } \\
\hline & & \multicolumn{3}{|c|}{ Clover } & \multicolumn{3}{|c|}{ wheat } & \multirow{2}{*}{\multicolumn{3}{|c|}{ Patata }} & \multicolumn{3}{|c|}{ Lettuce } \\
\hline & & No. & Red. & Red.\% & No. & Red. & Red.\% & No. & & Red.\% & No. & Red. & Red.\% \\
\hline \multirow{3}{*}{$\begin{array}{c}\text { Sharabass } \\
\text { village }\end{array}$} & Treatment 1 & 39.8 & $26.2 \mathrm{C}$ & 65.8 & 42.6 & $24.2 \mathrm{C}$ & 57 & 44.6 & $26.4 \mathrm{C}$ & 59 & 37.4 & $24.6 \mathrm{C}$ & 66 \\
\hline & Treatment 2 & 59.2 & $40.8 \mathrm{~B}$ & 68.9 & 61.0 & $38.6 \mathrm{~B}$ & 63 & 54.6 & $36.8 \mathrm{~B}$ & 67 & 51.2 & $34.8 \mathrm{~B}$ & 68 \\
\hline & Treatment 3 & 64.2 & $48.4 \mathrm{~A}$ & 75 & 67.6 & $48.8 \mathrm{~A}$ & 72 & 62.2 & $46.4 \mathrm{~A}$ & 75 & 63.4 & $48.6 \mathrm{~A}$ & 77 \\
\hline \multirow{3}{*}{$\begin{array}{l}\text { Faraskour } \\
\text { city }\end{array}$} & Treatment 1 & 45.8 & $28.4 \mathrm{C}$ & 62 & 39.0 & $20.4 \mathrm{C}$ & 52 & 31.2 & $18.6 \mathrm{C}$ & 58 & 30.2 & $19.8 \mathrm{C}$ & 66 \\
\hline & Treatment 2 & 55.0 & $36.4 \mathrm{~B}$ & 66 & 59.2 & $32.8 \mathrm{~B}$ & 55 & 51.0 & $34.4 \mathrm{~B}$ & 67 & 48.4 & $34.2 \mathrm{~B}$ & 71 \\
\hline & Treatment 3 & 65.0 & $44.8 \mathrm{~A}$ & 69 & 70.0 & $49.4 \mathrm{~A}$ & 71 & 69.0 & $46.2 \mathrm{~A}$ & 71 & 60.8 & $44.8 \mathrm{~A}$ & 74 \\
\hline \multirow{3}{*}{$\begin{array}{c}\text { El-Dahra } \\
\text { village }\end{array}$} & Treatment 1 & 42.8 & $24.6 \mathrm{C}$ & 57 & 43.4 & $22.8 \mathrm{C}$ & 51 & 43.2 & $26.4 \mathrm{C}$ & 61 & 38.2 & $22.6 \mathrm{C}$ & 59 \\
\hline & Treatment 2 & 63.0 & $38.2 \mathrm{~B}$ & 61 & 58.6 & $38.4 \mathrm{~B}$ & 66 & 50.4 & $34.8 \mathrm{~B}$ & 69 & 56.8 & $39.4 \mathrm{~B}$ & 66 \\
\hline & Treatment 3 & 73.0 & $48.4 \mathrm{~A}$ & 66 & 65.4 & $54.6 \mathrm{~A}$ & 83 & 74.2 & $56.4 \mathrm{~A}$ & 76 & 57.4 & $48.6 \mathrm{~A}$ & 85 \\
\hline \multirow{3}{*}{$\begin{array}{l}\text { El-Roda } \\
\text { city }\end{array}$} & Treatment 1 & 40.8 & $24.2 \mathrm{C}$ & 59 & 39.0 & $22.8 \mathrm{C}$ & 58 & 44.2 & $26.8 \mathrm{C}$ & 61 & 39.0 & $22.4 \mathrm{C}$ & 57 \\
\hline & Treatment 2 & 57.2 & $36.8 \mathrm{~B}$ & 64 & 57.4 & $34.6 \mathrm{~B}$ & 60 & 54.2 & $32.6 \mathrm{~B}$ & 60 & 56.8 & $34.4 \mathrm{~B}$ & 61 \\
\hline & Treatment 3 & 74.2 & $48.4 \mathrm{~A}$ & 65 & 60.4 & $48.4 \mathrm{~A}$ & 80 & 60.6 & $48.4 \mathrm{~A}$ & 80 & 69.6 & $48.8 \mathrm{~A}$ & 70 \\
\hline
\end{tabular}

Treatment 1= Superphosphate $15 \%+$ hydrated lime by percentage of 3:1

Treatment 2= Liquid of foliar fertilizer by dose of $1 \mathrm{~kg} / \mathrm{feddan}$

A : Signi. $B=n s$.

C=ns.

Treatment 3= Metaldhyde bait in special station

No $=$ Natural number of individuals (control).

Red. = Reduction of treated individuals.

Red. \%= Reduction Percentage.

Table 3: Effect of tested compounds on land snail Monacha cantiana oncertain field and vegetable crops at Faraskour district, Damietta Governorate through seasons of 2006/2007

\begin{tabular}{|c|c|c|c|c|c|c|c|c|c|c|c|c|c|}
\hline \multirow{3}{*}{ Location } & \multirow{3}{*}{$\begin{array}{c}\text { Tested } \\
\text { compounds }\end{array}$} & \multicolumn{12}{|c|}{ Number of affected individuals of Succinea Putris } \\
\hline & & \multicolumn{3}{|c|}{ Clover } & \multicolumn{3}{|c|}{ wheat } & \multicolumn{3}{|c|}{ Patata } & \multicolumn{3}{|c|}{ Lettuce } \\
\hline & & No. & Red. & Red.\% & No. & Red. & Red.\% & No. & Red. & Red.\% & No. & Red. & Red.\% \\
\hline \multirow{3}{*}{$\begin{array}{c}\text { Sharabass } \\
\text { village }\end{array}$} & Treatment 1 & 46.6 & $23.4 \mathrm{C}$ & 50.2 & 45.0 & $24.6 \mathrm{C}$ & 54.7 & 39.2 & $22.4 \mathrm{C}$ & 56.6 & 41.2 & $28.6 \mathrm{C}$ & 69 \\
\hline & Treatment 2 & 67.6 & $36.4 \mathrm{~B}$ & 53.8 & 59.0 & $32.8 \mathrm{~B}$ & 55.6 & 41.4 & $28.8 \mathrm{~B}$ & 69.6 & 53.2 & $32.4 \mathrm{~B}$ & 61 \\
\hline & Treatment 3 & 60.8 & $38.4 \mathrm{~A}$ & 63.2 & 67.8 & $44.4 \mathrm{~A}$ & 65.5 & 65.2 & $48.4 \mathrm{~A}$ & 74.0 & 71.0 & $46.6 \mathrm{~A}$ & 66 \\
\hline \multirow{3}{*}{$\begin{array}{l}\text { Faraskour } \\
\text { city }\end{array}$} & Treatment 1 & 44.6 & $20.2 \mathrm{C}$ & 45.3 & 42.0 & $21.6 \mathrm{C}$ & 51.0 & 39.2 & $20.4 \mathrm{C}$ & 52.0 & 40.6 & $21.8 \mathrm{C}$ & 54 \\
\hline & Treatment 2 & 60.8 & $28.4 \mathrm{~B}$ & 46.7 & 47.2 & $30.4 \mathrm{~B}$ & 64.4 & 46.0 & $29.4 \mathrm{~B}$ & 65.0 & 45.2 & $26.4 \mathrm{~B}$ & 58 \\
\hline & Treatment 3 & 75.4 & $48.6 \mathrm{~A}$ & 64.4 & 71.2 & $46.8 \mathrm{~A}$ & 66.0 & 69.2 & $46.4 \mathrm{~A}$ & 67.0 & 64.6 & $48.6 \mathrm{~A}$ & 75 \\
\hline \multirow{3}{*}{$\begin{array}{l}\text { El-Dahra } \\
\text { village }\end{array}$} & Treatment 1 & 46.6 & $24.4 \mathrm{C}$ & 52.3 & 47.2 & $24.4 \mathrm{C}$ & 52.0 & 48.4 & $28.8 \mathrm{C}$ & 59.0 & 41.0 & $26.4 \mathrm{C}$ & 64 \\
\hline & Treatment 2 & 64.6 & $36.8 \mathrm{~B}$ & 57.0 & 59.0 & $32.6 \mathrm{~B}$ & 55.0 & 54.4 & $30.4 \mathrm{~B}$ & 56.0 & 63.4 & $36.8 \mathrm{~B}$ & 58 \\
\hline & Treatment 3 & 71.2 & $48.4 \mathrm{~A}$ & 68.0 & 59.2 & $46.4 \mathrm{~A}$ & 79.0 & 70.2 & $49.4 \mathrm{~A}$ & 63.0 & 64.8 & $46.8 \mathrm{~A}$ & 72 \\
\hline \multirow{3}{*}{$\begin{array}{l}\text { El-Roda } \\
\text { city }\end{array}$} & Treatment 1 & 48.4 & $26.4 \mathrm{C}$ & 54.5 & 44.4 & $22.8 \mathrm{C}$ & 51.0 & 44.8 & $21.8 \mathrm{C}$ & 49.0 & 40.4 & $20.4 \mathrm{C}$ & 50 \\
\hline & Treatment 2 & 64.8 & $38.8 \mathrm{~B}$ & 60.0 & 64.6 & $36.4 \mathrm{~B}$ & 56.0 & 63.0 & $35.8 \mathrm{~B}$ & 57.0 & 66.6 & $38.6 \mathrm{~B}$ & 58 \\
\hline & Treatment 3 & 67.0 & $46.4 \mathrm{~A}$ & 69.3 & 63.2 & $48.4 \mathrm{~A}$ & 76.6 & 61.2 & $49.4 \mathrm{~A}$ & 81.0 & 62.2 & $46.2 \mathrm{~A}$ & 74 \\
\hline
\end{tabular}

Treatment 1= Superphosphate $15 \%$ + lime by percentage of 3:1

Treatment 2= Liquid of foliar fertilizer by dose of $1 \mathrm{~kg} / \mathrm{feddan}$

Treatment $3=$ Metaldhyde bait in special station

A : Signi.

$B=$ ns.

$\mathrm{C}=\mathrm{ns}$.

No.= Number of natural individuals ( control) 